\title{
TOWARDS THE INTEGRATION OF INDOORGML AND INDOORLOCATIONGML FOR INDOOR APPLICATIONS
}

\author{
L. Liu ${ }^{\mathrm{a}}$, S. Zlatanova ${ }^{\mathrm{a} *}$, Q. Zhu ${ }^{\mathrm{b}}, \mathrm{K} . \mathrm{Li}^{\mathrm{c}}$ \\ a Department of Urbanism, the Faculty of Architecture, Delft University of Technology, the Netherlands - (1.liu-1, s.zlatanova)@tudelft.nl \\ ${ }^{\mathrm{b}}$ Faculty of Geosciences and Environmental Engineering, Southwest Jiaotong University, Chengdu, Sichuan, China \\ - zhuq66@263.net \\ ${ }^{c}$ Department of Computer Science and Engineering, Pusan National University, South Korea - lik@ pusan.ac.kr
}

Commission IV, WG IV/4

KEY WORDS: IndoorGML, IndoorLocationGML, Location-Based Services, Indoor Navigation

\begin{abstract}
:
This paper introduces and compares two types of GML-based data standards for indoor location-based services, i.e., IndoorGML and IndoorLocationGML. By elaborating the advantages of the both standards and their data models, we conclude that the two data standards are complementary to each other. A jointed data model is presented to show the integration of the two standards. IndoorGML can supply subdivision of building for data of IndoorLocationGML, and the semantics of locations defined in IndoorLocationGML can be added to IndoorGML. By proposing two use cases, we take the initiative in attempting to combine the use of the two standards. The first case is to collect details from files of the two standards for an indoor path; the second one is to generate verbal directions for indoor guidance from files of the two standards. Some future work is given for further development, such as automatic integration of separate data from both standards.
\end{abstract}

\section{INTRODUCTION}

Indoor activities such as indoor navigation greatly rely on indoor spaces, i.e., regions with physical boundary or conceptual area without boundary (e.g., the dining area of a hall). Applications in the real world requires clear and sufficient space definitions, indoor navigation models (e.g., three-dimensional (3D) networks) simplifying building interiors and indoor routing/wayfinding systems (e.g., a pathfinding engine or signage system in the building) (Worboys, 2011; Lee and Kwan, 2005; Brown et al., 2013; Meijers et al., 2005; Becker et al., 2009; Thill et al., 2011; Boguslawski and Gold, 2010; Teo and Cho, 2016). Such information is partially organized in different data models and technical standards (Lee and Kwan, 2005; Worboys, 2011; Brown et al., 2013; Becker et al., 2009; Lee et al., 2014; Liu and Zlatanova, 2012). In addition, indoor navigation requires good expressions of location to be able to position one in the spaces or to navigate to a Point of Interest (PoI) with ease (Sithole and Zlatanova, 2016; Wijewardena et al., 2016; Kim and Li, 2016).

Crossing the current research one of the primary problems is to properly define and describe indoor spaces and locations, their relationships and other significant information (semantics, important attributes and geometry) (Worboys, 2011; Goetz and Zipf, 2011; Brown et al., 2013). Many explorations have been devoted in this direction. A typical and established piece of work for indoor modelling is IndoorGML (Lee et al., 2014), a data standard of Open Geospatial Consortium (OGC) about navigation network of indoor environments. It provides a concise expression of indoor navigation networks and related indoor spaces with their semantics. Another interesting study is an emerging Chinese data standard Multidimensional Indoor Location Information Model (IndoorLocationGML) focusing on indoor location, which aims to address urgent requirements on indoor location modelling (Zhu

\footnotetext{
${ }^{*}$ Corresponding author
}

et al., 2016). It proposes a framework that can manage both accurate and relative descriptions of indoor locations, enrich locations with semantics, and maintain topology of locations (e.g., distance, direction, order, etc.).

In this paper we investigate the two data standards of IndoorGML and IndoorLocationGML and aim to bridge the two standards to support more indoor navigation cases. The two standards concentrate on different aspects of indoor navigation, and thus they are complementary to each other for indoor navigation applications. Indoor $G M L$ focuses on navigation networks where pathfinding can be conducted, but IndoorLocationGML covers the issues related to indoor locations. Thus IndoorLocationGML aims for location-based services. In fact, the both standards can be used together for two main types of cases presented as follows:

- Support different indoor subdivision results.

- Generate verbal guidance for indoor navigation.

The rest of this paper is organized as follows: Section 2 will introduce the two data standards, Section 3 will present the uses of the both standards to each other. Section 4 will present two use cases by linking the two standards. This paper is closed with conclusions and some future work in Section 5.

\section{INDOORGML AND INDOORLOCATIONGML}

The two data standards are both presented with data model in $X M L$ schema (i.e., technical model) and based on Geography Markup Language (GML) (Portele et al., 2007). IndoorGML is based on connectivity of indoor spaces, and defines rules for navigable network; IndoorLocationGML focuses on ontology and application of indoor locations (e.g., absolute and relative locations, 
and direction). Therefore, the two data models can be complementary to each other on different aspects for indoor navigation. This section will introduce the key features of the two standards. Section 2.1 will present the overview of IndoorGML and section 2.2 will explain the data model of IndoorLocation $G M L$.

\subsection{IndoorGML}

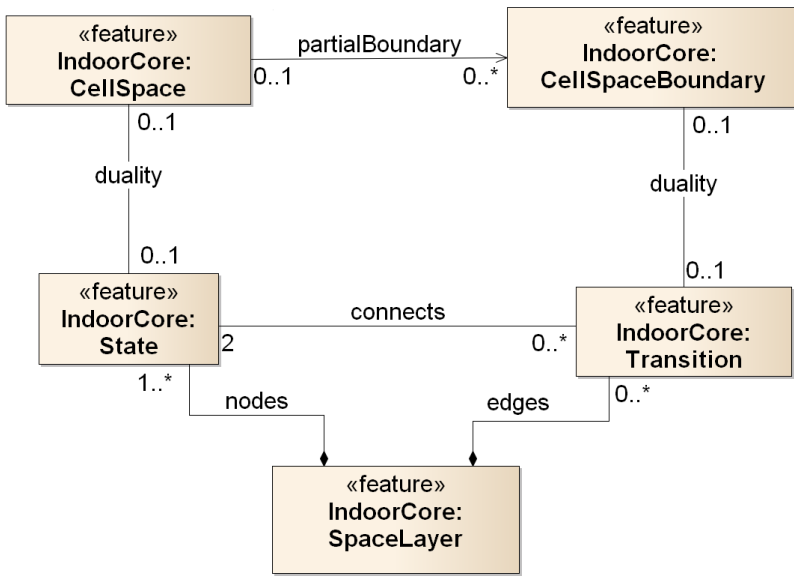

Figure 1. Main classes of Core module of IndoorGML (from Lee et al., 2014)

As mentioned before, Indoor $G M L$ is a data standard of $O G C$ that aims to define navigation network of indoor environments (Lee et al., 2014). It consists of an open data model and XML schema and it has two modules. The first one is Core module (see Figure 1). Basically it is about a topological representation of cellular spaces. These spaces and their boundaries correspond to CellSpace and CellSpaceBoundary. Other two primary classes of State and Transition link to CellSpace and CellSpaceBoundary according to Poincaré Duality (Whitney, 1932; González, 1984), respectively.

States and Transitions represent nodes and edges of the dual graph (Whitney, 1932) of indoor space. The two terms are the same as those in Multi-Layered Space Model (MLSEM) (Becker et al., 2009), i.e., another data model about indoor space information related to IndoorGML standard (Nagel, 2014). MLSEM proposes a semantic conceptual model for indoor spaces, and provides the geometric and topological representations of indoor spaces based on Poincaré Duality. Inspired by these indoor space representations in MLSEM, IndoorGML is designed as a common schema framework for indoor navigation applications (Lee et al., 2014).

The second module is Navigation which focuses on semantic features of indoor spaces. The Navigation module extends the Core module and defines semantics of spaces in the context of indoor navigation. The root classes about spaces are NavigableSpace and NavigableSpaceBoundary. They are subclasses of CellSpace and CellSpaceBoundary, respectively. The subclasses of NavigableSpace define all types of indoor spaces, such as NavigableSpace, GeneralSpace (e.g., common rooms), TransitionSpace (e.g., Hallway or Stairway) and AnchorSpace (e.g., main entrance linking indoors and outdoors). The Navigation module also represents indoor paths: classes of RouteNode, RouteSegment and Route. RouteNode associates to State, RouteSegment associates to Transition, and Route represents navigable paths. In such a way, space semantics (e.g., a TransitionSpace) can be reflected in a RouteNode.
In general, Indoor $G M L$ defines and expresses the network of indoor spaces (e.g., connectivity graph) and indoor paths. In addition, space semantic features are defined which can be used to enrich the semantics of indoor network and paths.

\subsection{IndoorLocationGML}
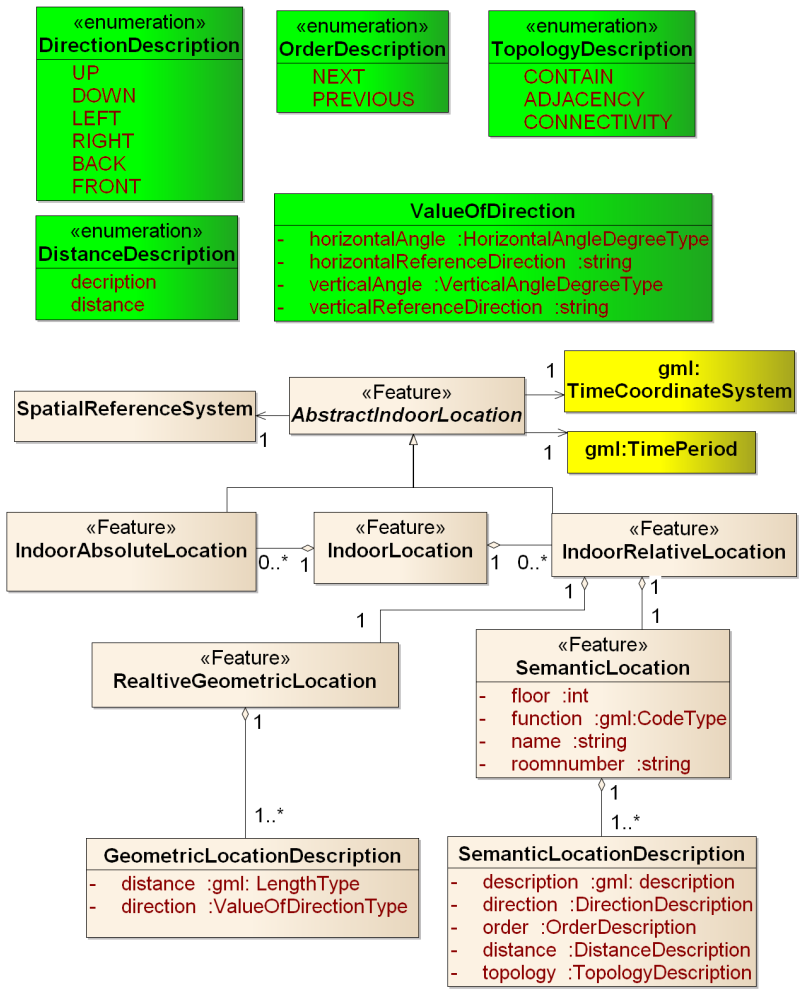

Figure 2. Main part of IndoorLocationGML (remade from Zhu et al., 2016)

As mentioned before, the developing Chinese standard IndoorLocationGML concentrates on definition and description of indoor location (Xiong et al., 2013; Zhu et al., 2016). The abstract class AbstractIndoorLocation stands for the general notion of indoor locations, which associates to three other classes: gml:TimeCoordinateSystem, gml:TimePeriod and SpatialReferenceSystem (Figure 2) . The first two are classes of GML Encoding Standard (Portele et al., 2007) and indicate the temporal features of AbstractIndoorLocation. SpatialReferenceSystem refers to an absolute or local coordinate system which provides measurement for instances of AbstractIndoorLocation.

Two subclasses of AbstractIndoorLocation, IndoorAbsoluteLocation and IndoorRelativeLocation, represent locations described in the coordinate system or with the relationships with other objects/locations, respectively. An IndoorLocation is composed of both IndoorAbsoluteLocation and IndoorRelativeLocation, which indicates the two types of measurement applied for one location. IndoorRelativeLocation have two subclasses: RelativeGeometricLocation and SemanticLocation (see Figure 2). The classes RelativeGeometricLocation contains the values of distance and direction (e.g., angles) to other reference objects; SemanticLocation provides descriptions of distance, direction (e.g., up, down, left and right), order (e.g., previous and next) and topology (e.g., connectivity and containment) to other reference locations. In general, the data model of IndoorLocationGML centers all classes on indoor locations (e.g., PoI). 
In order to present the relationships between the data models of the two standards, we integrate the core parts of the two models in one UML class diagram (see Figure 3). Two essential associations, IndoorLocation to CellSpace and RouteNode, are the key to integrate the two types of data model. The class IndoorLocation from IndoorLocationGML depicts any location in a space (i.e., CellSpace), and an RouteNode represents a space. In the association of CellSpace and IndoorLocation, the multiplicity on the IndoorLocation side is from 0 to multiple, which means a CellSpace may contain none or many IndoorLocation. Reversely, an IndoorLocation can associate only one CellSpace (i.e., the multiplicity 1). In the association of RouteNode and IndoorLocation, the multiplicity on the IndoorLocation side is none or many and that on the RouteNode side is none or one. It means that an RouteNode can either link to IndoorLocation or none, while an IndoorLocation can be either a location of a path (in RouteNode) or just an independent PoI.

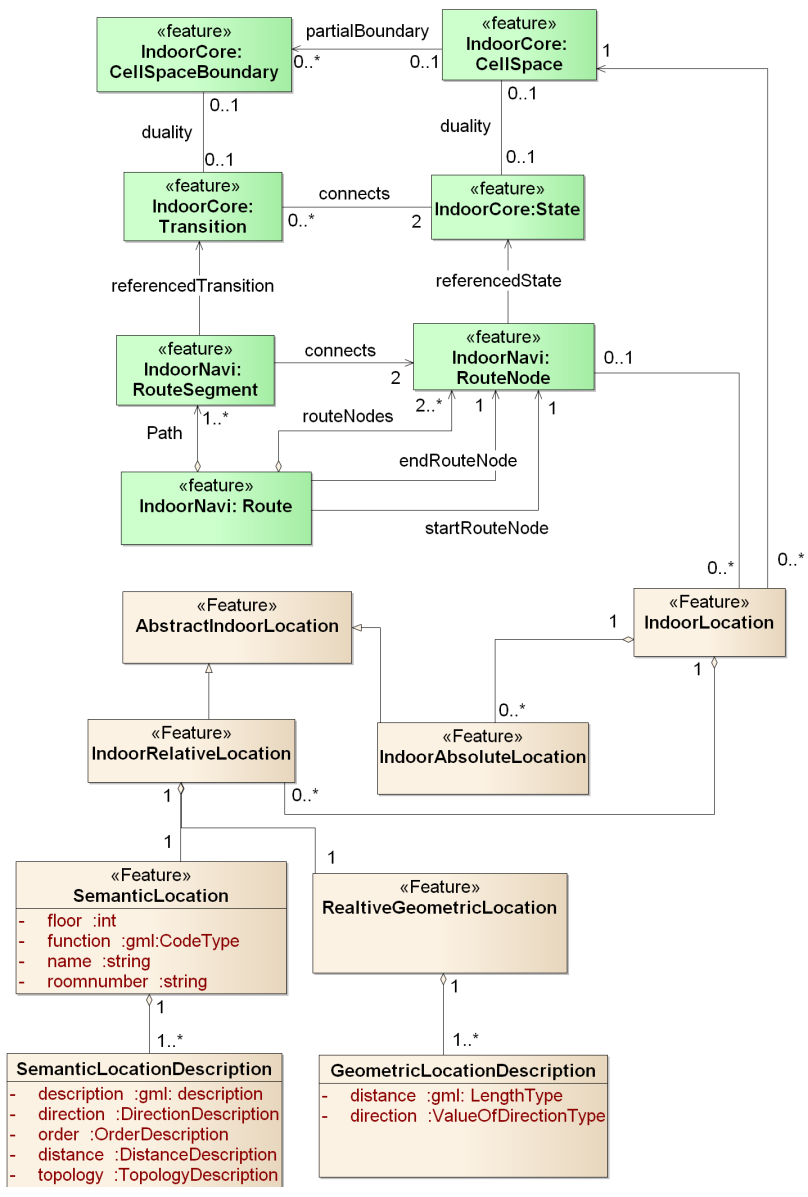

Figure 3. The jointed class diagram of IndoorGML and IndoorLocation GML

To sum up, IndoorLocationGML provides the complete description and measurement of indoor locations and models the relationships of indoor locations. Therefore, it can describe a sequence of locations and their relative relationships, such as locations in a path (e.g., to describe the motion from the previous location to the current location in distance and angle).

\section{COMBINED USE OF INDOORGML AND INDOORLOCATIONGML}

As addressed before, IndoorGML and IndoorLocationGML emphasize different aspects of indoor modelling. Thus the both standards can be use together for indoor navigation. Section 3.1 will show the use of IndoorLocationGML complementary to IndoorGML; and Section 3.2 presents the features of IndoorGML complementary to IndoorLocationGML.

\subsection{IndoorLocationGML complementary to IndoorGML}

Although in the Navigation module of IndoorGML semantics of spaces are provided, there is no definition of PoI. PoI can be frequently used in indoor navigation as position reference, especially when they can be added and deleted by users (see Figure 4). In this case, a PoI can be represented by instances of IndoorAbsoluteLocation and IndoorRelativeLocation. Coordinates of the PoI are reflected by the IndoorAbsoluteLocation and the semantics of the PoI (e.g., floor information and function) can be recorded in the IndoorRelativeLocation. Consequently, we can form a file containing the IndoorGML and IndoorLocationGML code snippets. For example, for a node the IndoorGML part includes the code of RouteNode and RouteSegment; and the IndoorLocationGML part contains the piece of IndoorAbsoluteLocation and/or IndoorRelativeLocation.

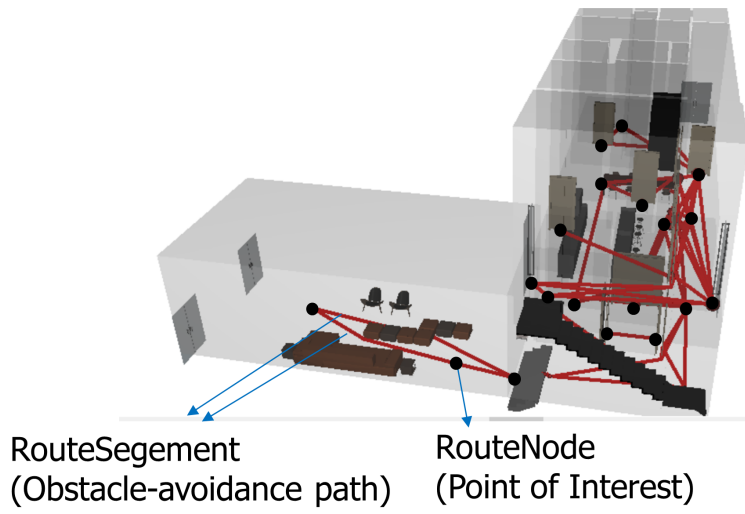

Figure 4. Example of RouteNode and RouteSegment which can be enriched by IndoorRelativeLocation instances

Another case is about TransitionSpace in IndoorGML. The class TransitionSpace refers to corridor, stair and subspaces of hallway (Lee et al., 2014). Generally it is about the horizontal and vertical connection parts of a building. However, the IndoorGML TransitionSpace does not specifically distinguish between horizontal and vertical spaces. Consequently, this ambiguity can be passed to the related instances of RouteNode. In this case, instances of IndoorRelativeLocation can be added to clarify whether the RouteNode related to TransitionSpace is horizontal or vertical.

Besides RouteNode, the semantics of RouteSegment can be also enriched by instances of IndoorRelativeLocation of IndoorLocationGML. For example, the relative position (up/down or left/right) of nodes on a RouteSegment can be depicted, and thus they can be perceived with ease. In addition, the room number can be also reflected with the IndoorRelativeLocation, which support the query about the room containing the RouteSegment. 
In summary, the IndoorGML may lack location information for some applications. The semantics defined and depicted in $I n-$ doorLocationGML can be used for the network described by IndoorGML. In this way, the semantics of nodes and edges of this network can be enriched.

\subsection{IndoorGML complementary to IndoorLocationGML}

IndoorGML can supply data for IndoorLocation GML when spacerelated information is required. Subdivisions of building can be reflected in IndoorGML files which is missing in IndoorLocationGML. A specific subdivision of building results in a number of indoor spaces. Besides the subdivision according to building structure (e.g., walls as boundary), other subdivisions can be achieved by using functionalities of indoor space (Krūminaitè and Zlatanova, 2014). According to (MLSEM) (Becker et al., 2009), the same building can be organized in different layers with different subdivision results. For example, one layer represented by a group of navigable spaces for a user and another layer consisting of the coverage area of a Wi-Fi transmitter in the whole building. For each layer, a network can be generated and described by State and Transition instances in an IndoorGML document. This information can be integrated with an IndoorLocationGML file.

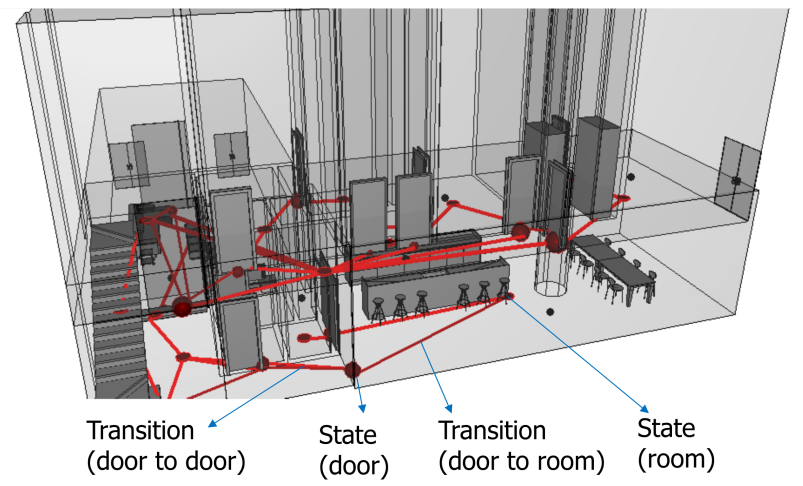

Figure 5. Example of subdivision result of a residence building.

Figure 5 shows a case that doors are regarded as space. Normally a thin door is considered as $2 \mathrm{D}$ surface and thus the connectivity graph of spaces is constructed for rooms. But in IndoorGML a thick Door with depth attribute is also considered as space. In the presented example, the connectivity graph is about rooms and doors. This connectivity graph can be depicted by instances of State and Transition. A State can represent a Room or a Door. In this way, three types of Transition can be identified: 'door-todoor', 'door-to-room' and 'room-to-room'. Such semantic information from the subdivision can be added to the related IndoorLocation GML file.

Space semantics in the Navigation module of IndoorGML can also be passed into an IndoorLocationGML file. For example, an instance of State is assigned with semantics of the related space (e.g., TransitionSpace). Corresponding indoor locations organized in the IndoorLocationGML file can be assigned the semantics according to their relationships to these State instances.

In a word, IndoorGML carries the subdivision result of buildings, which can flexibly describe different indoor networks. This information can be added to IndoorLocationGML and semantics of the space containing indoor locations could be clarified.

\section{USE CASES}

Previously the features of IndoorGML and IndoorLocationGML have been explained. It is feasible to combine these two data standards for indoor navigation applications. By integrating the two types of data model, examples of indoor navigation that are benefited by this combination could be:

\section{Case 1: Path detail enrichment}

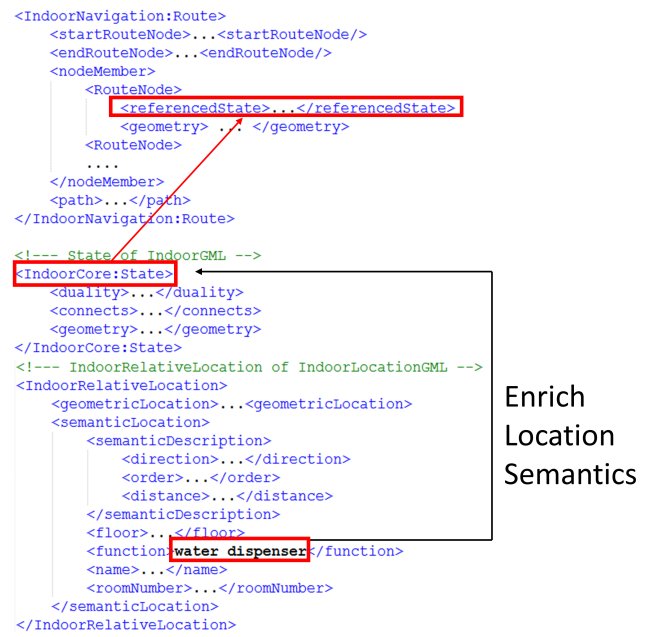

Figure 6. IndoorLocationGML provides detailed location semantics for Route instances

IndoorGML and IndoorLocationGML both contain path-related information. The class Route of IndoorGML maintains indoor paths. By using IndoorLocationGML, other characteristics of this path can be revealed. For example, each RouteNode of a Route can be enriched with nearby locations (Wi-Fi active points or smoke detectors) described in IndoorRelativeLocation. In such cases, it is easy to count the resource locations that a path involves, such as query points, kiosks and water dispensers in the path. In Figure 6 each RouteNode of a Route has related State. An IndoorRelativeLocation instance supplies this State with the location semantics (the function 'water dispenser'). This combination provides diverse information about a path for different users.

A path can be also conveyed by IndoorLocation GML if the path is represented by a sequence of locations. The path contains both the coordinates and the sequence of these locations. Semantic information of these locations are also conveyed with the class SemanticLocation. In addition, IndoorGML can supply space subdivision result for the path (see Figure 7). A State related to CellSpace follows the subdivision contained in partialbound$e d B y$. This information is also supplied to the related instance of IndoorRelativeLocation.

In practice, the integration of separate datasets from both standards can be performed in two possible ways. The first one is to generate a unified data document according to the jointed UML data model. The other one is to develop a parser which can acquire the data of the two types of dataset. This paper discusses only the integration of the two standards on the conceptual level. The implementation of such integration is left to the future work.

\section{Case 2: Generation of verbal directions}




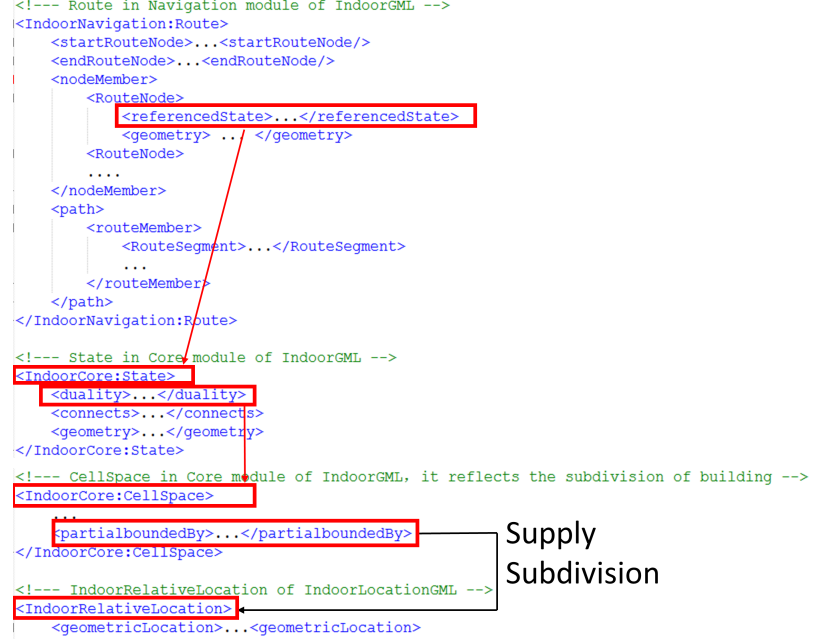

Figure 7. IndoorGML supplies subdivision result for IndoorRelativeLocation instances

Verbal directions are used to improve user's understanding of path derived from indoor navigation (Russo et al., 2014). For a given path, verbal directions regarding guidance for users can be generated by using IndoorGML and IndoorLocationGML together. IndoorGML can represent indoor path in spaces as RouteNode and RouteSegment. Specifically, space of rooms and doors are represented by RouteNode; and a Route can present an abstact path in 'room-to-room', 'door-to-door' and 'room-door-room' styles. However, RouteNode does not include its direction and distance information to other nodes. Besides, RouteNode does not necessarily include all POI in the related space. For instance, in Figure 8 instances of RouteNode are N1, N2, N3 and N4. N1 and $\mathrm{N} 3$ are specific nodes which stand for Office 1 and Corridor1, respectively. $N 2$ and $N 4$ are the reference nodes of two doors. By IndoorGML, this Route of N1-N2-N3-N4 is a 'room-door-room' path. The point of interest $P O I 1$, representing the location of a pillar, cannot be indicated by the class RouteNode.

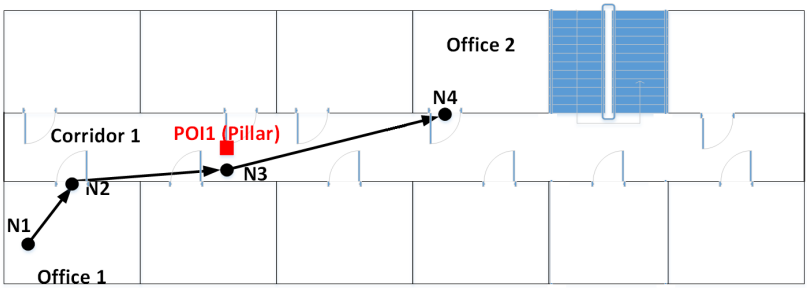

Figure 8. Example of a path in a floor

Semantics from IndoorLocationGML supports directional/topologica and geometric details to $N 1, N 2, N 3$ and $N 4$. IndoorLocation $G M L$ can supplement detailed location semantics and relative positions between the locations, such as $N 2$ is next to N1, and N2 is in the front-right of N1. The unity of two standards can give an complete overview on the path (see Figure 8 ). The geometric locations of N1,N2,N3 and N4 are on the same floor. According to the related IndoorLocation $G M L$ document, $N 2$ is next to $N 1$ in 3 meters $(\mathrm{m})$ and on right of it in the direction of 60 degree. Similarly the relative position of $N 3$ to $N 2$ and that of $N 4$ to $N 3$ can be decoded from the IndoorLocationGML document. Specially, the geometric location POII can be used to assist the user to better locate her/his movement: the user can confirm the direction between $N 2$ and $N 3$ when she/he perceives POII. Finally, we can obtain verbal directions for this path shown below:

'This path is on the same floor, started from office 1, through one corridor and ended at office 2. Each step is assumed 70cm.'

'Started from the location (N1) and turn front-right. After 4 steps you can move out through the door ( $\mathrm{N} 2)$ and then turn right on the corridor'

'Keep this direction, after 6 steps you must see a pillar on your left.'

'Turn front-left, after 10 steps you could see a door (N4) on the left. Then you arrive at office 2 .'

\section{CONCLUSION}

This paper presents the possibility to use two indoor-related standards for indoor applications, i.e., IndoorGML and IndoorLocation GML. We briefly present and compare the two standards. They focus on aspects of indoor modelling. IndoorGML focuses on indoor navigation networks and space representation of different subdivisions. While IndoorLocationGML concentrates on a complete representation and description of indoor locations. For indoor navigation, the two standards are complementary to each other: the subdivision information carrying by IndoorGML can be added to IndoorLocationGML, and the semantics of locations defined in IndoorLocationGML can be complementary to IndoorGML.

In this paper we propose first ideas to a conceptual integration of the two indoor standards. On the level of data model, the essential class of IndoorLocation GML named IndoorLocation associates with two IndoorGML classes, i.e., CellSpace (Core module) and RouteNode (Navigation Module). Based on the relationships, two types of applications are considered: 1) supply subdivision details (e.g., indoor spaces) from IndoorGML to IndoorLocationGML datasets; and 2) supplement location details (e.g., direction, distance and all PoI) from IndoorLocationGML to IndoorGML datasets. We also present the related use cases considering the combined use of the two standards as initiatives. The result shows it is feasible to utilize the two data standards together for path detail enrichment and verbal directions generation.

In the future, the combination of the two standards can be further explored, such as to incorporate IndoorLocationGML semantics for different subdivisions depicted by IndoorGML (e.g., functional subdivision). Another work is to create a jointed format to integrate data from the two standards, which can facilitate their use for realistic applications. Based on the jointed format, automatic integration of separate data from both standards can be investigated. Tests need to be conducted to generate data files with the two standards for the same dataset. In this way, applications can be developed to load and visualize data of indoor networks and locations.

\section{ACKNOWLEDGEMENTS}

Building data of this research are provided by Bentley System Inc.. Special thanks are also extended to Mark Anderson, Alain Lapierre and Mark Smith of Bentley System Inc.. We also thanks anonymous reviewers for their helpful comments and constructive suggestions. 


\section{References}

Becker, T., Nagel, C. and Kolbe, T., 2009. A multilayered space-event model for navigation in indoor spaces. In: J. Lee and S. Zlatanova (eds), 3D Geo-Information Sciences, Lecture Notes in Geoinformation and Cartography, Springer Berlin Heidelberg, pp. 61-77.

Boguslawski, P. and Gold, C., 2010. Euler operators and navigation of multi-shell building models. In: T. Neutens and P. Maeyer (eds), Developments in 3D Geo-Information Sciences, Springer Berlin Heidelberg, Berlin, Heidelberg, pp. 116

Brown, G., Nagel, C., Zlatanova, S. and Kolbe, T., 2013. Modelling $3 \mathrm{~d}$ topographic space against indoor navigation requirements. In: J. Pouliot, S. Daniel, F. Hubert and A. Zamyadi (eds), Progress and New Trends in 3D Geoinformation Sciences, Lecture Notes in Geoinformation and Cartography, Springer Berlin Heidelberg, pp. 1-22.

Goetz, M. and Zipf, A., 2011. Extending OpenStreetMap to indoor environments. CRC Press, pp. 51-61. doi:10.1201/ b11647-7.

González, J. R. M., 1984. Elements of algebraic topology.

Kim, J.-S. and Li, K.-J., 2016. Location k-anonymity in indoor spaces. GeoInformatica 20(3), pp. 415-451.

Krūminaitè, M. and Zlatanova, S., 2014. Indoor space subdivision for indoor navigation. In: Proceedings of the Sixth ACM SIGSPATIAL International Workshop on Indoor Spatial Awareness, ACM, pp. 25-31.

Lee, J. and Kwan, M., 2005. A combinatorial data model for representing topological relations among $3 \mathrm{~d}$ geographical features in microspatial environments. International Journal of Geographical Information Science 19(10), pp. 1039-1056. doi=http://dx.doi.org/10.1080/13658810500399043.

Lee, J., Li, K., Zlatanova, S., H.Kolbe, T., Nagel, C. and Becker, T., 2014. OGC IndoorGML Version 1.02. Document No.14$005 \mathrm{r} 4$.

Liu, L. and Zlatanova, S., 2012. A semantic data model for indoor navigation. In: Proceedings of the Fourth ACM SIGSPATIAL International Workshop on Indoor Spatial Awareness, ISA '12, ACM, New York, NY, USA, pp. 1-8.

Meijers, M., Zlatanova, S. and Pfeifer, N., 2005. 3d geoinformation indoors: structuring for evacuation. In: Proceedings of Next generation 3D city models, pp. 21-22.

Nagel, C., 2014. Spatio-semantic modelling of indoor environments for indoor navigation. $\mathrm{PhD}$ thesis, Technische Universit"at Berlin.

Portele, C., Cox, S. J. D., Daisey, P., Lake, R. and Whiteside, A., 2007. OpenGIS Geography Markup Language (GML) Encoding Standard Version 3.2.1. Document No.07-036.

Russo, D., Zlatanova, S. and Clementini, E., 2014. Route directions generation using visible landmarks. In: Proceedings of the Sixth ACM SIGSPATIAL International Workshop on Indoor Spatial Awareness, ISA '14, ACM, New York, NY, USA, pp. 1-8.
Sithole, G. and Zlatanova, S., 2016. Position, location, place and area: An indoor perspective. ISPRS Annals of Photogrammetry, Remote Sensing and Spatial Information Sciences III-4, pp. 89-96.

Teo, T.-A. and Cho, K.-H., 2016. Bim-oriented indoor network model for indoor and outdoor combined route planning. Advanced Engineering Informatics 30(3), pp. 268-282.

Thill, J.-C., Dao, T. H. D. and Zhou, Y., 2011. Traveling in the three-dimensional city: applications in route planning, accessibility assessment, location analysis and beyond. Journal of Transport Geography 19(3), pp. 405 - 421. Special Issue : Geographic Information Systems for Transportation.

Whitney, H., 1932. Non-separable and planar graphs. Transactions of the American Mathematical Society 34, pp. 339 - 362.

Wijewardena, G. G., Vasardani, M. and Winter, S., 2016. Towards indoor localization and navigation independent of sensor based technologies. In: Proceedings of the Eighth ACM SIGSPATIAL International Workshop on Indoor Spatial Awareness, ISA '16, ACM, New York, NY, USA, pp. 19-26.

Worboys, M., 2011. Modeling indoor space. In: Proceedings of the 3rd ACM SIGSPATIAL International Workshop on Indoor Spatial Awareness, ISA '11, ACM, New York, NY, USA, pp. 1-6. doi=http://doi.acm.org/10.1145/2077357.2077358.

Xiong, Q., Zhu, Q., Zlatanova, S., Huang, L., Zhou, Y. and $\mathrm{Du}, \mathrm{Z}$., 2013. Multi-dimensional indoor location information model. ISPRS - International Archives of the Photogrammetry, Remote Sensing and Spatial Information Sciences ISPRS Acquisition and Modelling of Indoor and Enclosed Environments 2013 (XL-4/W4), pp. 45-49.

Zhu, Q., Li, Y., Xiong, Q., Zlatanova, S., Ding, Y., Zhang, Y. and Zhou, Y., 2016. Indoor multi-dimensional location gml and its application for ubiquitous indoor location services. ISPRS Int. J. Geo-Inf. 5(12), pp. 220. 